Recibido: 11/09/2019 --- Aceptado: 04/12/2019 --- Publicado: 15/07/2020

\title{
DISEÑO, PRODUCCIÓN E IMPLEMENTACIÓN DEL AMBIENTE DE APRENDIZAJE BASADO EN WEB, AABW
}

\section{DESIGN, PRODUCTION AND IMPLEMENTATION OF LEARNING ENVIRONMENT BASED ON WEB, LEBW}

Rómulo Andrés Gallego Torres ${ }^{1}$. Colegio Mayor de Cundimarca. Colombia. andresgallegot@gmail.com

\section{RESUMEN}

El trabajo que aquí se presenta, forma parte de la necesidad de contribuir a la educación en ciencias a través de las TIC. En este sentido, se diseñó un Ambiente Basado en la WEB, el cual presenta en su estructura una estrategia didáctica de andamiaje de tipo metacognitivo, el cual, se diseñó en el contexto de aprendizaje de ciertos contenidos de tipología textual en relación con las Ciencias Naturales. La investigación planteó un diseño cuasiexperimental de tipo factorial $2 \times 3$, con grupos previamente conformados pertenecientes a dos cursos regulares de un colegio privado de la ciudad de Bogotá. La experiencia consistió en exponer a los grupos de estudiantes a un Ambiente Basado en la WEB para el fortalecimiento de la comprensión lectora y las competencias científicas. Los resultados de esta investigación aportan a docentes, terapeutas y en general a la Comunidad educativa elementos frente al uso de un andamiaje metacognitivo en un Ambiente Basado en la WEB, como estrategia didáctica para el desarrollo de los procesos metacognitivos y el logro de aprendizaje. Dicha estrategia integrada junto con el estilo cognitivo en la dimensión DFI de los estudiantes podría predecir el éxito académico de los aprendices cuando interactúan con este tipo de ambientes.

PALABRAS CLAVE: ambientes basados en la WEB - andamios metacognitivos juicios metacognitivos - dimensión DFI - Metacognición - LMS - competencia lectora.

\section{ABSTRACT}

The work presented here is part of the need to contribute to science education through ICT. In this sense, a WEB-based environment was designed, which presents in its structure a didactic strategy of scaffolding of a metacognitive type, which was designed in the context of learning certain contents of textual typology in relation to the Sciences Natural. The research raised a quasi-experimental design of type $2 \times 3$,

1 Rómulo Andrés Gallego Torres: Master in Information Technology Applied to Education. Professor Colegio Mayor de Cundimarca. Bogota Colombia.

andresgallegot@gmail.com 
with previously formed groups belonging to two regular courses of a private school in the city of Bogotá. The experience consisted of exposing groups of students to a WEB-based environment for strengthening reading comprehension and scientific skills. The results of this research contribute to teachers, therapists and in general to the Educational Community elements regarding the use of a metacognitive scaffolding in a WEB-based environment, as a teaching strategy for the development of metacognitive processes and learning achievement. Such an integrated strategy along with the cognitive style in the students' DFI dimension could predict the academic success of apprentices when interacting with such environments.

KEYWORDS: WEB-based environments - metacognitive scaffolding - metacognitive judgments - DFI dimension - Metacognition - LMS - reading competence.

\section{DESIGN, PRODUÇÃO E IMPLEMENTAÇÃO DO AMBIENTE DE APRENDIZADO BASEADO NA WEB, AABW}

\section{RESUMO}

O trabalho aqui apresentado, faz parte da necessidade de contribuir à educação em ciências através das TIC. Neste trabalho, foi criado um Ambiente Baseado na WEB, este apresenta na sua estrutura uma estratégia didática de andaimes do tipo metacognitivo, o que foi criado no contexto de aprendizado de alguns conteúdos de tipología textual relacionados com as Ciências Naturais. A pesquisa propõe um design quase experimental de tipo fatorial $2 \times 3$, com grupos previamente escolhidos pertencentes a dois cursos regulares de uma escola privada da cidade de Bogotá. A experiência consistiu em expor aos grupos de alunos ao Ambiente Baseado na WEB para o fortalecimento da compreensão da leitura e as competências científicas. Os resultados desta pesquisa trazem a professores, terapeutas e a toda Comunidade educativa em geral, elementos diante do uso de andaimes de tipo metacognitivo em Ambientes Baseados na WEB, como estratégia didática para o desenvolvimento dos processos metacognitivos e conquista do conhecimento. Dita estratégia integrada com o estilo cognitivo na dimensão DFI dos alunos poderia prever o sucesso acadêmico dos aprendizes quando interactuam com este tipo de ambientes.

PALAVRAS CHAVE: ambientes baseados na WEB - andaimes metacognitivos julgamentos metacognitivos - dimensão-DFI - Metacognição - LMS - competência leitora.

\section{Cómo citar el artículo:}

Gallego Torres, R. A. (2020). Diseño, producción e implementación del ambiente de aprendizaje basado en web, AABW. [Design, production and implementation of learning environment based on web, LEBW]. Revista de Comunicación de la SEECI, (52), 119-147. doi: http://doi.org/10.15198/seeci.2020.52.119-147

Recuperado de http://www.seeci.net/revista/index.php/seeci/article/view/617 


\section{INTRODUCCIÓN}

La dinámica cambiante del mundo actual propone diferentes desafíos, que requieren de personas capaces de desarrollar procesos de pensamiento que les permitan abordar, comprender y resolver diferentes problemas. En una sociedad donde la gestión de la información es privilegiada, es de gran importancia promover el desarrollo de la competencia lectora, enfatizando el carácter interpretativo y constructivo que requiere este ejercicio; Dado que leer implica además de reconocer e interpretar diferentes sistemas de signos, reflexionar sobre nuestro propio pensamiento, tomar conciencia de los procesos involucrados en la realización de una tarea, a fin de fortalecer el pensamiento crítico, flexible y reflexivo creativo (Cerchiaro, Paba, y Sánchez, 2011) (Cantillo, De la Hoz y Cerchiaro, 2014)

En este sentido, es importante comprender que existen dos procesos de lectura interdependientes: a) los procesos de reconocimiento de los signos y caracteres y b) el proceso macro de comprensión de lectura; según Snowling y Hulme:

(...) la competencia lectora va más allá del dominio de las habilidades de reconocimiento de palabras o la fluidez de lectura, y también es necesario un dominio estratégico de los procesos cognitivos de alto nivel involucrados en la comprensión lectora para lograr una interpretación y comprensión profundas del texto (en Gundín, Fidalgo y Robledo, 2012).

De esta manera, existe un vínculo estrecho entre la comprensión lectora y la actividad metacognitiva. Entendiendo esto, como la capacidad de un individuo de tener control sobre sus pensamientos y mantener la conciencia de los mismos, con el fin de integrar su conocimiento a las demandas concretas de un mundo cambiante, donde la gestión de la información y la capacidad de rápida respuestas efectivas son privilegiadas (De Corte, 1999).

De acuerdo con lo anterior para el ámbito nacional, se incluyó a partir del segundo semestre de 2014 en el $11^{\circ}$ conocimiento aplicado a estudiantes de último grado de todo el país, el componente de lectura crítica, cuyo objetivo es favorecer una transformación orientada a fortalecer la evaluación de capacidades de razonamiento interpretativo y lógico de un texto y para evitar el conocimiento declarativo. Este componente implica el desarrollo de diferentes competencias por parte del alumno, para que éste logre acercarse críticamente a un texto, debe en primer lugar, comprender las unidades locales que se expresan. En segundo lugar, debe integrar esa información para darle un sentido global al texto. $Y$, en tercer lugar, una vez que se han superado las dos etapas anteriores, debe adoptar una postura crítica ante el texto, reflexionando sobre su contenido (ICFES, 2013).

Sin embargo, tomando como referencia a Herrera (2005), este plantea, desarrollar habilidades para pensar de manera crítica y creativa, ser flexible en las formas y métodos de observar una realidad y dar respuestas que sean efectivas en su aplicación; Habilidades incrustadas en la metacognición son un gran reto educativo, en ese sentido, Tamayo (2016) dice: La Metacognición "todavía se considera 
fundamental no sólo en los procesos de enseñanza y de aprendizaje, sino, además, en la constitución del pensamiento crítico".

Por otro lado, la competencia de la comprensión lectora en pruebas internacionales ha mostrado grandes dificultades; Teniendo en cuenta el desempeño de los estudiantes de los ocho países latinoamericanos, estos aún están lejos de los estándares de calidad definidos por la Organización para la Cooperación y el Desarrollo Económico (OCDE) que a través de la prueba PISA evalúa las competencias de los estudiantes en matemáticas, lectura y ciencias naturales. Para el caso específico de lectura, los resultados arrojados por los estudiantes colombianos evaluados en el año 2012 informan que:

el $51 \%$ de los estudiantes no alcanzó el nivel básico de competencia, y el 31\% fue colocado en el nivel 2. Esto significa que tres de cada diez estudiantes colombianos pueden detectar uno o más fragmentos de información dentro de un texto; además, reconocen la idea principal, entienden las relaciones y crean significados dentro de los textos que requieren inferencias simples, y pueden comparar o contrastar desde una característica única del texto. En los niveles 5 y 6 solo hay 3 de cada mil jóvenes que pueden hacer inferencias múltiples, realizar cruces y contrastes detallados y precisos; Demuestran una comprensión amplia y detallada de uno o más textos, y hacen una evaluación crítica de un texto cuyo contenido no es familiar (ICFES, 2013, p. 9).

Para 2015, Colombia mejoró las estadísticas del SUS en el campo de la comprensión lectora, aunque todavía estamos rezagados con respecto a los países del primer mundo, este año las cifras no se ubicaron a la par de países como México, Turquía, entre otros, alcanzando 425 puntos, 22 más que en 2012. El resultado más problemático es que el $43 \%$ de los menores colombianos no exceden los estándares mínimos de la OCDE en esta área (OCDE, 2016).

Por lo tanto, las reflexiones se plantean desde el campo académico, de manera concreta desde la actividad docente con estudiantes de secundaria y universitarios destacando las dificultades que presentan los jóvenes en el desarrollo de la competencia de lectura. Generando interés por conocer las variables que afectan el proceso de comprensión lectora en formato digital.

La metacognición, como una actividad mental mediante la cual otros procesos o estados mentales se convierten en objeto de reflexión, constituye una variable importante en el proceso de comprensión lectora, según Cerchiaro, Paba y Sánchez (2011) y Cantillo, De la Hoz y Cerchiaro (2014). Los componentes metacognitivas clave se distinguen de regular a la comprensión de lectura; $k$ conocimiento del propósito de la lectura y autorregulación de la actividad mental para lograr ese objetivo; Informa que los lectores menos competentes muestran un conocimiento insuficiente del propósito de la tarea y de las estrategias del lector, así como una capacidad deficiente para supervisar su propio proceso de comprensión.

De acuerdo con Paba y González (2014) establecieron la relación entre la actividad metacognitiva y la comprensión lectora en estudiantes de secundaria, encontrando 
que la actividad metacognitiva en la muestra es nula $y$, por lo tanto, el nivel de comprensión lectora es bajo.

Desde esta perspectiva y para fomentar el desarrollo de estrategias metacognitivas, Molenaar y Sleegers (2010) Van de Pol, Volman, Oort y Beishuizen (2015) proponen el uso de andamios metacognitivos, que tienen la función de gestionar y regular los procesos cognitivos. Este tipo de apoyo permite al sujeto elevar metas de aprendizaje consistentes con los intereses y el tiempo disponible, de la misma manera que lo ayuda a supervisar el avance contra la meta propuesta y a reflexionar sobre los resultados obtenidos, en busca de estrategias de reorientación que se hayan demostrado sin éxito en el logro de los aprendizajes deseados.

Otra variable relacionada con el desempeño de los estudiantes en la comprensión de lectura en formato digital es el estilo cognitivo, concepto propuesto por Witkin en 1948; y según Hederich et al. (2013) e Hidalgo y Olaya (2016) es el estilo cognitivo en la independencia o dependencia de campo (DFI) que establece diferencias entre los sujetos, relacionados con la capacidad de reestructuración cognitiva, procesamiento de la información, competencias Interpersonal y motivaciones entre las polaridades de dos sujetos: los llamados independientes y dependientes del campo. Estas diferencias afectan el proceso de aprendizaje, el logro del aprendizaje individual y la forma en que los estudiantes acceden al conocimiento en entornos computacionales (López-Vargas y Hederich-Martínez, 2011), (López, Ibáñez y Chiguasuque, 2014).

En este contexto, Korthauer y Koubek (2009) establecen el efecto del estilo cognitivo de los sujetos y su relación con las tareas desarrolladas en entornos hipertextuales, descubriendo que los estudiantes independientes de campo presentan más exactitud en sus tareas de aprendizaje en comparación con los dependientes de campo, lo que permite deducir que estos estudiantes tienen mayores habilidades para analizar y sintetizar información. Con respecto al desempeño de los estudiantes que dependen del campo, se descubrió que no utilizaron correctamente las ayudas explícitas en un entorno hipermedia y que requieren más tiempo para realizar la tarea.

Tomando como referencia el marco anterior estos objetivos de investigación para diseñar un entorno basado en la web, implementan dentro de su estructura un andamio metacognitivas, que a través de activadores objetivos metacognitivos, promueven el desarrollo o la capacidad metacognitiva de los estudiantes $y$, en consecuencia, posiblemente, a mejorar la comprensión lectora de los mismos cuando interactúan con textos en formato digital.

\section{INVESTIGAR OBJETIVOS}

\subsection{Objetivo general}

Crear y validar un apoyo de tipo metacognitivo en un entorno de aprendizaje basado en la web para el desarrollo de procesos metacognitivos en comprensión de 
lectura y el logro de aprendizaje en ciencias naturales, minimizando las diferencias entre los estudiantes con diferentes estilos cognitivos.

\subsection{Objetivos específicos}

- Diseñar e implementar un apoyo metacognitivo en un entorno de aprendizaje basado en la web para el desarrollo del proceso metacognitivo en la comprensión lectora en estudiantes de secundaria.

- Investigación sobre la efectividad del apoyo metacognitivo en entornos de aprendizaje basados en la web en el desarrollo de competencias científicas en individuos con diferentes estilos cognitivos.

- Identifique las características de los procesos metacognitivos de los estudiantes con diferentes estilos cognitivos en la dimensión DFI, al interactuar con un apoyo metacognitivo en un entorno de aprendizaje básico en la web.

\section{ENFOQUE DEL PROBLEMA}

El propósito de este estudio es analizar diferentes aspectos relacionados con los procesos metacognitivos en la comprensión lectora para fomentar la reflexión e implementación de estrategias que faciliten el logro de los objetivos de aprendizaje y la resolución de problemas en los estudiantes (Jiménez, 2004). Desde esta perspectiva, se diseñó un entorno basado en la WEB, que presenta en su estructura una estrategia didáctica de apoyo de tipo metacognitivo; este apoyo fue diseñado en el contexto del aprendizaje de cierto tipo de contenido textual en relación con las ciencias naturales. En consecuencia, además del desarrollo de la capacidad metacognitiva, se espera que la interacción con este entorno computacional favorezca a los estudiantes en relación con el logro de aprendizaje esperado.

Los resultados de esta investigación contribuyen a los maestros, terapeutas y, en general, a los elementos de la comunidad educativa contra el uso del apoyo metacognitivo en un entorno basado en la WEB, como estrategia didáctica para el desarrollo de procesos metacognitivos y el logro del aprendizaje. Esta estrategia integrada junto con el estilo cognitivo en la dimensión DFI de los estudiantes podría el éxito académico de los aprendices cuando interactúan con este tipo de entornos.

Del mismo modo, los resultados de esta investigación con respecto al diseño y desarrollo de entornos de aprendizaje basados en la web podrían ser considerados mediante la formación de educadores en tecnología de la información y comunicación. Como constituyen un recurso importante para los estudiantes de diferentes estilos cognitivos porque favorece el desarrollo de su capacidad metacognitiva en la comprensión lectora y, por lo tanto, las posibilidades de lograr los académicos esperados. En este sentido, el problema central de esta investigación se puede resumir a través de la siguiente pregunta:

¿Cuál es el efecto del apoyo metacognitivo en el logro del aprendizaje de ciencias naturales en estudiantes de secundaria que interactúan con entornos de aprendizaje basados en la web? 


\section{ALGUNAS REFERENCIAS TEÓRICAS}

\subsection{Metacognición en entornos de aprendizaje basados en web-AABW}

La revolución de las comunicaciones en las sociedades posindustriales ha llevado a un cambio en las formas de producción, distribución y consumo: el énfasis del circuito productivo reside en el llamado trabajo inmaterial (creación y producción de bienes simbólicos; por decir, información, conocimiento y know-how cuya infraestructura se basa en diferentes formas de expresión del lenguaje). No solo las formas de producir cambios: también cambian las formas de percepción y expresión, lo que tiene un profundo impacto en la configuración del conocimiento $y$, por supuesto, afecta las formas tradicionales de entender la pedagogía. La presencia de actores en el proceso La educación del aprendizaje ya no es un requisito y la centralidad del libro de texto y el maestro se están moviendo para dar forma a nuevas modalidades de comunicación pedagógica.

En este sentido, la educación virtual o e-learning contribuye a un mejor aprendizaje porque en muchas áreas se considera que la educación externa, a la del aula es más propicia teniendo en cuenta la revolución del conocimiento, esto ha generado que los alumnos desarrollen autónomamente aprendizaje, además de ser considerados nativos digitales y tener acceso a la información de los diferentes dispositivos a su alcance, debe pensar en una estrategia metacognitiva en el campo de las TIC. Primero, han transformado el proceso cognitivo y, como lo define, se ha generado un nuevo tipo de inteligencia, la inteligencia distribuida que se define como "la inteligencia no es una propiedad atribuida a las mentes de los individuos, sino que se distribuye entre las personas, y entre las personas y las herramientas físicas y los sistemas simbólicos" (Herrero y Brown, 2010), esto ha llevado a nuevos paradigmas y nuevas formas de enseñanza y aprendizaje, como el conectivismo, que intenta demostrarlo a través de las redes de aprendizaje, las redes sociales, el autoaprendizaje y entornos personales de aprendizaje, además del uso de las TIC, los estudiantes pueden producir su conocimiento de forma autónoma, trascendiendo su construcción del aula virtual y ser evaluados por ellos y sus compañeros, simplemente dejando al maestro como mediador de lo que aprendí ( Gallego Torres, 2017).

En segundo lugar, debe considerarse que, gracias al uso de entornos computacionales, según Karl Stephen (Sierra, Carrascal y Buelvas, 2014) se puede dividir en dos tipos: Sistema de contenedores, que es cómo se han utilizado comúnmente los entornos de aprendizaje virtual ,son repositorios de información preestablecidos y centrados en el entorno, y los recursos ofrecidos por las diferentes plataformas, además del sistema de contenido donde los usuarios proporcionan y comparten información y contenido, logrando una apropiación del curso por parte de los estudiantes que participan en el proceso y no solo como receptores de conocimiento, sino no, como pro-cosumidores, eso significa que consumen y producen la información necesaria para lograr el objetivo de aprendizaje propuesto por el curso, esto se logra con la articulación del contenido propuesto por los 
estudiantes y con las actividades, evaluaciones y comentarios de los docentes, que utilizan herramientas asincrónicas como foros, blogs, etc.

Para esta investigación se utilizó una mezcla de los estilos porque en el Moodle se utilizó como depósito de contenidos, pero las evaluaciones temáticas se realizaron sugiriendo a los alumnos que realicen comentarios a través de los foros publicados en cada unidad, esto permitiría a los estudiantes participar en su proceso de aprendizaje y que generan la capacidad de regular el aprendizaje, buscando lo que se llama vidas combinadas, los estudiantes interactúan mezclando su realidad entre la cara y lo virtual, están conectados, inmersos en las diferentes plataformas existentes, Internet, Smart TV, etc., logrando crear entornos de aprendizaje personales reales logrando un aprendizaje autónomo (Sierra, Carrascal y Buelvas, 2014).

Por último, pero no menos importante, existen estrategias de mediación metacognitiva en entornos virtuales, ya que este tema es central en el proyecto de investigación, pero son las estrategias metacognitivas, según Isabel Sierra en su tesis doctoral "Estrategias de mediación metacognitiva en entornos convencionales y virtuales: influencia en los procesos de autorregulación y aprendizaje autónomo en los estudiantes", los definen como:

[...] Son el conjunto de acciones orientadas a conocer las propias operaciones y procesos mentales [...] Son aplicados por el sujeto, durante y después de sus procesos de aprendizaje y tienen el objetivo de optimizar sus ejecuciones de manera consciente. (Sierra, 2010).

En otras palabras, son las estrategias que los estudiantes realizan Mayer (Sierra, 2010), se pueden dividir en tres categorías; A) que permiten la planificación de acciones cognitivas, B) que permiten percibir el avance de la meta y C) las que modifican el plan o ajustan la acción según sea necesario.

Se puede definir que los juicios metacognitivos son las preguntas que surgen tanto de los estudiantes como de sus maestros que permiten evaluar sus procesos de aprendizaje, estos pueden ser abstractos, ¿tiene claras las características de un texto expositivo?, o está listo para demostrar su comprensión de este texto, sus estándares se basan en el contexto social o son creados por él, esto favorece la generación de pensamiento crítico en los estudiantes haciendo que su aprendizaje regule y guíe hacia el camino que conducirá al autoaprendizaje.

Existen varias investigaciones en las que su base de investigación es la AABW, tomarán los ejemplos en Colombia, para comenzar esta investigación se lleva a cabo en la Universidad piloto de Colombia titulada "Estrategia didáctica en los entornos de aprendizaje basados en la web: Regulación de andamios", realizada por Nicolás García D. En 2014, la prueba se realizó a 50 estudiantes universitarios y buscó el desarrollo de habilidades autorreguladas de aprendizaje de resolución de problemas matemáticos a través del logro del aprendizaje esperado, en esta investigación los resultados fueron positivos porque mostraron que el apoyo de regulación implementado en el AABW impactado de manera positiva en el logro del aprendizaje, 
también evidenció que no. Hubo diferencias significativas en los resultados causados por el estilo cognitivo y terminaron recomendando el desarrollo de AABW propicio para la regulación del aprendizaje, ya que fomentan la reflexión y el control de su propio proceso de aprendizaje, ayudando positivamente en la motivación de los estudiantes (García, 2015).

En segundo lugar, analizó la investigación realizada por Isabel Sierra P. Calidad del aprendizaje y procesos de metacognición y autorregulación en entornos virtuales y duales en la educación superior, la investigación se basó en establecer la relación entre las Estrategias de enseñanza metacognitiva en entornos virtuales y los niveles de desarrollo del aprendizaje autónomo y como esto motivó las estrategias de autorregulación en el aprendizaje de estudiantes universitarios en este caso del sexto semestre de la Facultad de Educación de la Universidad de Córdoba en Colombia, este estudio utilizó la tipología de entornos virtuales, que los divide en:

a. Entorno virtual orientado al desarrollo instrumental y uso de recursos para la documentación.

b. Entorno virtual orientado al desarrollo de competencias, fortalecimiento de modelos de trabajo y aprendizaje de procedimientos.

c. Entorno virtual orientado al desarrollo de actividades de representación, estrategias de aprendizaje cognitivo y metacognitivo.

d. Entorno virtual orientado al desarrollo de procesos de colaboración, participación y gestión de reuniones de socialización de ideas y proyectos (2011, pp. 83-85).

\subsection{Diferencias de aprendizaje individual}

Dentro de cada grupo académico hay evidencia de diferencias entre los estudiantes que lo constituyen, algunas de estas diferencias se deben a factores asociados con el género, las creencias religiosas, el origen étnico o los aspectos económicos, sociales o culturales; Estas diferencias se enmarcan dentro del campo emocional, social o cognitivo, a partir de esto se elabora el concepto de estilo. (Lozano, 2006).

\section{Los estilos}

se consideran como un conjunto de características que las personas han relacionado con sus comportamientos en aquellas áreas donde actúan y se relacionan. Esto es útil para analizar las diferentes formas de acción. Los estilos conforman la categorización de los diversos comportamientos que hacen que cada uno persona particular y original (Renes y Martínez, 2016).

El estilo se define como el conjunto de regularidades características de los sujetos que determinan su comportamiento y se caracteriza por ser: 1) diferenciador, en la medida en que establece características distintivas entre las personas; 2) relativamente estable en cada individuo; 3 ) Integrador de las diferentes dimensiones del sujeto, y 4) neutral, es decir, que no se debe valorar, en términos absolutos, un estilo por encima de otro (Hederich-Martínez, 2007). 
La segunda categoría son los estilos de aprendizaje, que se refieren a las preferencias que los estudiantes tienen cuando se trata de procesar información o frente a la realización de una tarea de aprendizaje (Alonso, C., Gallegos, D., y Honey, P., 1997), (Cerchiaro, Paba y Sánchez, 2011).

En el campo educativo, en algunas ocasiones las dos categorías descritas anteriormente se utilizan en términos de estilo de aprendizaje y estilo cognitivo; Sin embargo, estos conceptos son diferentes, para diferenciar entre estos dos conceptos es útil utilizar el modelo propuesto por Curry, en 1987, que propone "El modelo de la cebolla" (gráfico 1).

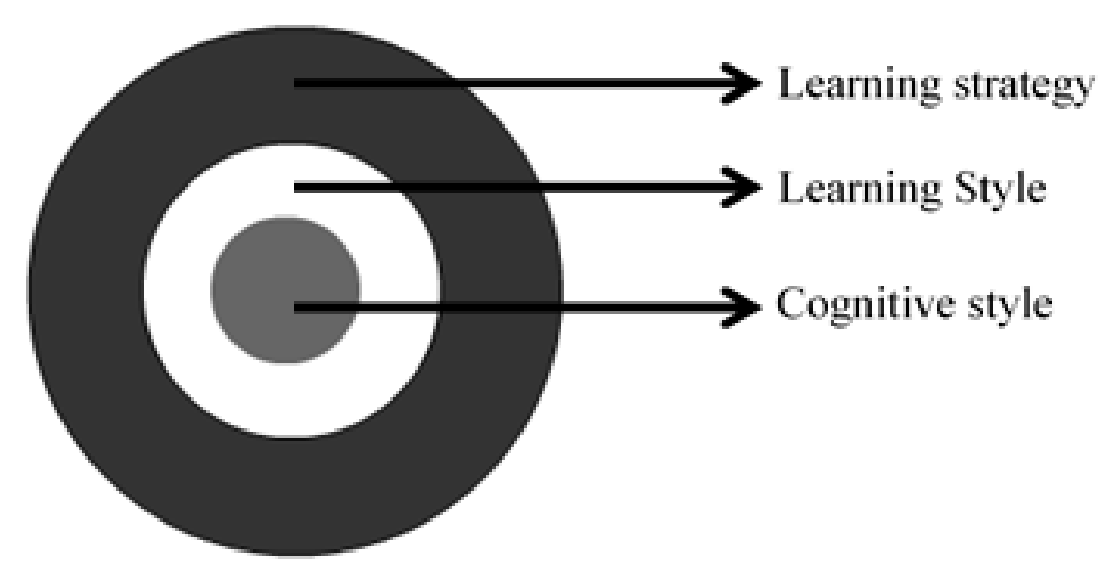

Gráfico 1: modelo de cebolla al curry.

Fuente: López, Hederich y Camargo (2011).

\subsection{El estilo cognitivo en la dimensión DFI}

En Hederich (2013), el estilo cognitivo que posiblemente ha sido más estudiado es el estilo de dependencia-independencia de campo (DFI) propuesto por Witkin (1948), esta dimensión determina las diferencias entre los sujetos relacionados con la capacidad de reestructuración cognitiva, información procesamiento, competencias interpersonales y motivaciones entre dos polaridades de sujetos: los llamados dependientes independientes y de campo.

Estas diferencias en las asignaturas afectan directamente el proceso de aprendizaje, el rendimiento académico individual y cómo acceder al conocimiento en entornos computacionales (Hederich-Martínez y Camargo-Uribe, 2018), las asignaturas categorizadas como independientes del campo tienen mayores habilidades de reestructuración cognitiva, lo cual es evidente en su capacidad de desenmascarar figuras simples en figuras complejas, procesar información de manera analítica, situación que favorece el tiempo para profundizar los conceptos previamente adquiridos y establecer relaciones entre estos. Del mismo modo, tienen estrategias que facilitan el almacenamiento y la recuperación de la información, muestran preferencias hacia el trabajo individual y tienen una motivación intrínseca. (López-Vargas y Hederich-Martínez, 2011), (García, 2015), (Hederich-Martínez, 2007). 
En el otro polo se ubican temas llamados dependientes del campo, también llamados sensibles al medio ambiente, que tienen capacidades más bajas en relación con la reestructuración cognitiva, procesan la información de manera global, limitando la posibilidad de hacer inferencias y análisis profundos de la información, están orientados hacia el grupo trabajan y están extrínsecamente motivados.

La siguiente tabla establece algunas características de dependiente de campo (DC) e independiente de campo.

Tabla 1. Características de IC y DC (Hederich-Martínez, 2007).

\begin{tabular}{|l|l|}
\hline \multicolumn{1}{|c|}{ Dependencia de campo } & \multicolumn{1}{c|}{ Independencia de campo } \\
\hline $\begin{array}{l}\text { Percepción global: son difíciles de separar } \\
\text { las partes de la información de su contexto }\end{array}$ & $\begin{array}{l}\text { Percepción articulada: percibir las partes } \\
\text { como elementos separados del campo. }\end{array}$ \\
\hline $\begin{array}{l}\text { Personas pasivas que necesitan abundante } \\
\text { apoyo social. }\end{array}$ & $\begin{array}{l}\text { Personas activas que trabajan con poca } \\
\text { motivación externa, su confianza está en } \\
\text { sus referencias internas. }\end{array}$ \\
\hline $\begin{array}{l}\text { Se presentan fácilmente a la autoridad } \\
\text { correspondiente en parte porque tienen } \\
\text { poca iniciativa, la crítica a menudo tiene un } \\
\text { gran impacto en ellos }\end{array}$ & $\begin{array}{l}\text { Tener una actitud de liderazgo, sus } \\
\text { acciones se basan más en su escala de } \\
\text { valores internos que en la autoridad } \\
\text { externa. }\end{array}$ \\
\hline $\begin{array}{l}\text { Su aprendizaje es más eficiente a través de } \\
\text { métodos expositivos. }\end{array}$ & $\begin{array}{l}\text { Su aprendizaje es más eficiente a través } \\
\text { de métodos de descubrimiento. }\end{array}$ \\
\hline $\begin{array}{l}\text { Necesitan condiciones de motivación } \\
\text { extrínsecas. }\end{array}$ & $\begin{array}{l}\text { Aprende mejor en condiciones } \\
\text { intrínsecamente motivadas }\end{array}$ \\
\hline $\begin{array}{l}\text { Es fácil para las relaciones interpersonales; } \\
\text { prefieren trabajar en grupo }\end{array}$ & $\begin{array}{l}\text { Las relaciones interpersonales son a } \\
\text { menudo difíciles, casi siempre prefieren } \\
\text { el trabajo individual. }\end{array}$ \\
\hline
\end{tabular}

Fuente: Hederich-Martínez, 2007.

Dentro de esta misma categorización se ubican sujetos intermedios (INT), que tienen características de los dos grupos de extremos (DC) e (IC) (Min y Reed, 1994).

Estas características tienen una alta incidencia en el comportamiento de los sujetos en los campos académicos; De hecho, se ha observado que los estudiantes de campo independientes superan en el rendimiento académico a sus compañeros Sensibles al medio ambiente.

\subsection{Estilo cognitivo y logros de aprendizaje en AABW}

En el contexto educativo, el estilo cognitivo es un factor muy importante porque determina cómo las personas perciben, almacenan, transforman y procesan la información que tiene una influencia directa en el logro del aprendizaje (García, 2015), (López Ó., 2008).

Alrededor de esto, la AABW o los llamados AABC (entornos de aprendizaje basados en computadora) han generado una gran expectativa en términos de mejorar el logro del aprendizaje ya que tienen ventajas sobre las diferencias en el 
estilo cognitivo, logrando un mayor respeto por el ritmo de aprendizaje, de la misma manera, favorecen diferentes formas de interacción social y permiten el acceso a la información desde cualquier punto de navegación, combinando diferentes representaciones del dominio del aprendizaje (gráfico, videos, sonidos, animaciones, etc.). Sin embargo, hay varios estudios que han cuestionado la efectividad de AABC (Spiro y Jehng, 1992), (Tergan, 1997).

Con respecto al tema López (2008), sugiere que la $A A B C$ requiere una mayor autonomía por parte del estudiante en lo que respecta a la regulación del aprendizaje; mientras tanto, Astleitner y Leuner (Hermann y Detlev, 1995) afirman que la $A A B C$ tiene básicamente tres dificultades; el primero se refiere a la gran cantidad de información que se presenta a los estudiantes, que puede conducir a la distancia del propósito de aprendizaje, utilizando parte del tiempo en situaciones de poca importancia y dejando de atender a lo verdaderamente importante, la segunda dificultad está relacionada a la estructura misma de la $A A B C$ o AABW porque pueden causar desorientación espacial, perdiendo de vista los archivos herederos entre los contenidos tratados, lo que dificulta la estructuración del conocimiento en sí, ya que la tercera dificultad informa el gran esfuerzo cognitivo requerido por el estudiante para lograr la organización del volumen de información que se le presenta.

A modo de síntesis, el estilo cognitivo se define como el conjunto perceptual, características analíticas, intelectuales, sociales y afectivos que hacen que las personas procesan la información de una manera diferente, es muy estable durante toda la vida y es neutral, es decir, no se posible decir que un estilo es mejor que otro (Hederich-Martínez, 2007), sin embargo, es posible afirmar que afecta directamente el logro del aprendizaje.

El estilo cognitivo de cada asignatura, junto con las características de $A A B C$ o $A A B W$, genera un cierto grado de desigualdad para todos los estudiantes, ya que hace que los estudiantes independientes del campo, dada su estructura cognitiva, obtengan un mejor rendimiento académico frente a los dependientes del campo, en estudiantes

\subsection{Metacognición y comprensión de textos}

De acuerdo con el marco, la componente metacognitive juega un papel clave en la comprensión de lectura. Se pueden identificar dos componentes metacognitivos clave que intervienen en la regulación de la comprensión de lectura; se compromete con el propósito de la lectura (para lo que se lee) y la autorregulación de la actividad mental para lograr ese objetivo (cómo leer), lo que requiere controlar la actividad mental de cierta manera y dirigirla hacia un objetivo específico.

Ambos aspectos están estrechamente relacionados; la forma en que lee y regula la actividad mental mientras lee está determinada por el propósito que está buscando al leer. No lee un texto de la misma manera para pasar el tiempo y explicar el contenido de una clase; Tampoco el mismo ejercicio mental si se lee para identificar las ideas principales, para buscar el mejor título de un texto, para deducir 
conclusiones o para hacer un juicio crítico de los contenidos de este (Cantillo, De la Hoz y Cerchiaro, 2014) Brown (1986) señala que la metacognición en la comprensión de textos implica el conocimiento de cuatro variables y la forma en que interactúan para facilitar la comprensión.

Las variables involucradas están en primer lugar; el texto que incluye las características (estructura, nivel de dificultad, grado de familiaridad) de los materiales de lectura que afectan su comprensión y su memoria. En segundo lugar, se encuentra la tarea, que incluye los requisitos de almacenamiento y recuperación de información, que generan una ejecución por parte del lector. En tercer lugar, podemos encontrar las estrategias, que son las actividades que el lector implementa para almacenar y recuperar la información. Como y último lugar están las características del lector, su habilidad, su nivel de motivación y otros estados y atributos personales que influyen en la comprensión.

Del mismo modo, la metacognición en el proceso de lectura implica procesos de control y autorregulación. Tales procesos, de acuerdo con Baker y Brown (1984) son los siguientes:

1. Aclarar los propósitos de la lectura.

2. Aclarar las demandas de la tarea.

3. Identifique los aspectos importantes del mensaje contenido en el texto.

4. Centra la atención en las ideas principales y no en los detalles.

5. Monitorear las actividades realizadas para determinar el nivel de comprensión.

6. Participar en actividades generadoras de preguntas para determinar si se están cumpliendo los objetivos previos a la lectura.

7. Tomar medidas correctivas cuando se detecten dificultades de comprensión.

8. Evitar interrupciones y distracciones.

\section{MÉTODO}

La investigación planteó un diseño experimental de tipo factorial $2 \times 3$, con grupos previamente formados que pertenecen a dos cursos regulares de una escuela privada en la ciudad de Bogotá. La experiencia consistió en exponer a los grupos de estudiantes a un entorno basado en la WEB para fortalecer la comprensión lectora y las competencias científicas.

El entorno basado en la WEB contiene tres (3) unidades de aprendizaje relacionadas con la tipología textual en textos científicos: 1] texto descriptivo, 2] texto narrativo y 3 ] texto expositivo. Al final de cada unidad de estudio, todos los estudiantes presentaron individualmente una evaluación de la comprensión lectora a través de textos científicos. En total, se obtuvieron tres (3) evaluaciones para cada estudiante, que se promediaron al final del estudio.

Antes y después de la experiencia de los estudiantes realizan la prueba ESCOLA es un instrumento que evalúa la comprensión de la lectura objetivo de analizar los posibles problemas en la estrategia de la lectura de los niños de 8 a 13 años, es 
recomendable que esta prueba se complementa con la observación directa de al lado de un juicio serio por los $p$ adres 0 maestros y con las pruebas que miden el rendimiento y la comprensión de lectura de los niños.

La función principal es identificar las brechas en la conciencia de lectura y aprender sobre las estrategias que los estudiantes usan en el proceso de lectura.

Además, se utilizó la prueba de la figura enmascarada, que es el instrumento utilizado para determinar el estilo cognitivo en sus dimensiones 1 . Independencia, 2. Dependencia e 3. Intermedio, fue elaborado por H. Witkin en 1950, pero fue estandarizado para el contexto colombiano por Hederich \& Camargo en 1999 y su nivel de confiabilidad es 0.91 .

\subsection{Descripción del medio ambiente}

Presentación del curso: se retoman los datos relacionados con el curso, nombre, enfoque pedagógico, público objetivo, duración e intensidad horaria.

a. Comprensión de lectura: aquí se dará una definición clara y comprensible del tema de estudio.

b. Infografía de tipos de texto: diseñada para recordar fácilmente el tema de estudio

c. Evaluación inicial: el cuestionario ESCOLA se implementó para conocer la condición de los estudiantes al comienzo del curso.

d. Foro de comentarios: se implementó para la comunicación asincrónica con los estudiantes, en la cual los estudiantes pudieron completar todas las preguntas relacionadas con el curso.

La segunda parte consta de 3 unidades de estudio seleccionadas para la investigación, estas fueron: texto descriptivo, texto narrativo y texto expositivo, cada unidad tiene las siguientes partes:

a. Presentación: aquí está el contenido temático de la unidad, además de tener un texto explicativo tiene un video que explica el tipo de texto estudiado en cada una de las unidades.

b. Ejemplo: aquí hay un texto del tipo que la unidad está desarrollando y una prueba de control de lectura y con la cual se analizará el conocimiento del alumno.

c. Otros ejemplos: aquí encontrará más ejemplos del tipo de texto estudiado con sus respectivas pruebas para reforzar lo que han aprendido.

d. Complemente lo que aprendió: aquí hay una infografía que describe y explica el tipo de texto tratado, y se les pide a los estudiantes que comenten en el foro de la unidad.

e. Evaluación: es el espacio donde identificamos lo que hemos aprendido a través de una evaluación de las competencias de lectura. En él revisaremos el nivel de comprensión y análisis de los temas tratados en esta reunión.

f. Reflexión: aquí podría interactuar con los otros participantes y resolver preguntas relacionadas con la dinámica de la unidad estudiada. 
La tercera y última parte es la despedida, se realiza la evaluación final que permite evidenciar la movilización de procesos metacognitivos, a través de la finalización del cuestionario Escola (posttest).

El apoyo metacognitivo visualizado por el grupo de estudio se realizó al azar a través de los siguientes ensayos metacognitivos.

\subsubsection{Al comienzo del curso}

¿Cuánto sabes sobre el tema de estudio?

¿Qué tan importante crees que es el contenido de este tema de estudio?

¿Qué tan competente se siente para aprender esta unidad?

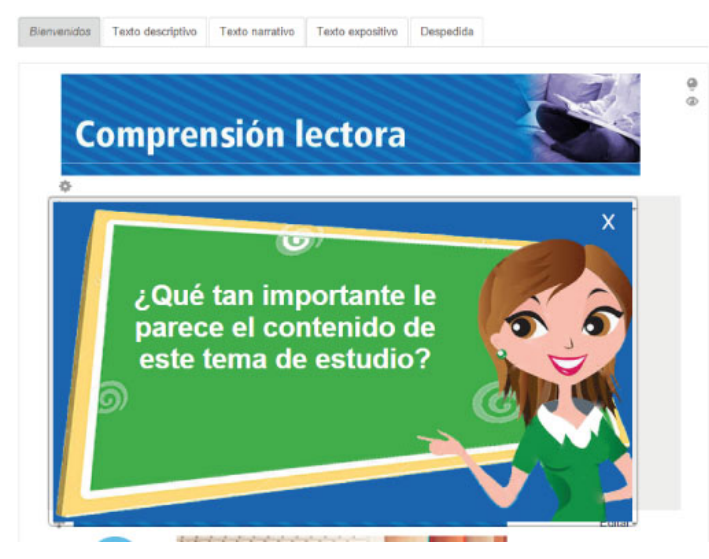

Imagen 1: Activador metacognitivo inicial.

\section{Unidad 1:}

¿Has sido claro en los conceptos de lectura?

¿Eres capaz de responder a una evaluación?

¿Puedes hacer una síntesis?

¿Estas entendiendo?

¿Te das cuenta si estás siguiendo lo que propusiste?

¿Han sido efectivas sus estrategias?

\section{Unidad 2:}

¿Podrías decir las características del texto narrativo? ¿Podría dar una definición sobre el texto narrativo? ¿Tienes claras las características de un texto narrativo?

\section{Unidad 3:}

¿Podría decir las características del texto expositivo? ¿Podría dar una definición sobre el texto expositivo? ¿Son claras las características de un texto de exposición? 


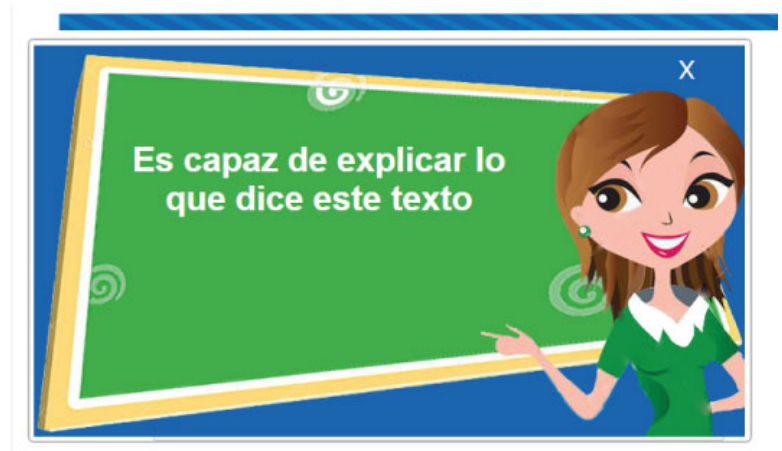

Después de leer el texto realice la prueba de comprensión

Imagen 2: imagen de CA de la unidad.

Antes de iniciar pruebas y evaluaciones

¿Estás listo para responder las preguntas?

¿Eres capaz de probar tu comprensión de este texto?

¿Estás listo para demostrar tu comprensión de este texto?

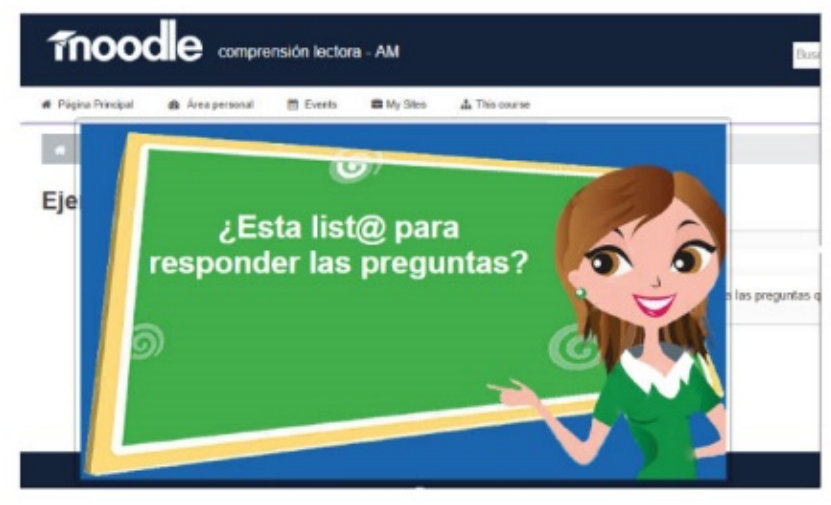

Imagen 3: Activador Prequiz.

\subsection{Propuesta metodológica. Diseño, producción e implementación de la AABW}

Para lograr los objetivos se diseñó el curso, se utilizó la metodología recomendada en las guías del libro para el diseño e implementación de cursos virtuales, la colección Sistema nacional de innovación educativa con uso de TIC, Ministerio de Educación Nacional, que nos brinda La hoja de ruta de un curso virtual, se divide en 5 etapas:
a. Diagnóstico y planificación
b. Diseño pedagógico
c. Producción de recursos y modelado educativo.
d. Montaje en plataforma
e. Despliegue y actualización 
Diagnóstico y planificación de la etapa 1: es el primer paso en la creación de un curso virtual que se caracteriza por el estudio que identifica las necesidades y los objetivos de aprendizaje que se atienden, además del análisis del contexto y el público objetivo.

Esta etapa se realiza para tener información veraz sobre las necesidades de los estudiantes frente al proceso de capacitación, los contenidos y los niveles de calidad.

Diseño pedagógico de la etapa 2: contiene y define todos los elementos, además de las estrategias didácticas del curso, el resultado de esto es el guion instructivo, el número de unidades y los contenidos temáticos se definen además de los diferentes OVA a ser utilizado, en este punto se diseñan las actividades y evoluciones relacionadas con el tema de estudio.

Etapa 3 producciones de recursos y modelado educativo: dos actividades principales se llevan a cabo en esta etapa; El primero es la producción de todos los contenidos y recursos que se desarrollan para el curso, el segundo es definir los caminos de aprendizaje que en la etapa anterior se han establecido.

Etapa 4 montaje de la plataforma: en esta etapa, la implementación del curso se lleva a cabo en la plataforma LMS en nuestro caso Moodle V-2.9.1, esta etapa incluye el desarrollo, el diseño de las diferentes piezas gráficas, su estructura y su funcionamiento.

Etapa 5 implementación y actualización: es la etapa final donde se desarrollan las estrategias diseñadas para el curso virtual y el curso se lleva a cabo con los estudiantes, después de un análisis del desarrollo del curso, basado en los diferentes comentarios proporcionados por los participantes. puede realizar una Actualización de contenido textual y multimedia, evaluaciones y diferentes actividades que contiene el curso.

\section{Descripción del entorno Aprendizaje basado en la web (curso virtual)}

Para el desarrollo de nuestra investigación se diseñó un curso virtual montado en la plataforma Moodle, por su facilidad de uso y para que pueda ser reutilizado. El curso consta de 3 partes, la primera es la bienvenida donde el estudiante será recibido y tiene las siguientes partes:

1. Presentación del curso: en esta parte se proporcionarán los datos relacionados con el curso, nombre, enfoque pedagógico, público objetivo, duración e intensidad de tiempo.

2. Comprensión de lectura: aquí se dará una definición clara y comprensible del tema de estudio.

3. Infografía de los tipos de texto: se diseñó para recordar fácilmente el tema de estudio.

4. Evaluación inicial: La prueba ESCOLA se implementó para conocer la condición de los estudiantes al comienzo del curso. 
5. Foro de retroalimentación: se implementó para la comunicación asincrónica con los alumnos, en este se pudo realizar todas las preguntas relacionadas con el curso.

La segunda parte consta de 3 unidades de estudio seleccionadas para la investigación, estas fueron: texto descriptivo, texto narrativo y texto expositivo, cada unidad tiene las siguientes partes:

1. Presentación: aquí está el contenido temático de la unidad, además de tener un texto explicativo tiene un video que explica el tipo de texto estudiado en cada una de las unidades.

2. Ejemplo: aquí hay un texto del tipo que la unidad está desarrollando y una prueba de control de lectura y que analizará el conocimiento del estudiante.

3. Otros ejemplos: aquí encontrará más ejemplos del tipo de texto estudiado con su respectivo examen para reforzar lo que aprendieron.

4. Complemente lo que aprendió: Aquí hay una infografía que describe y explica el tipo de texto tratado, y se les pide a los estudiantes que comenten en el foro de la unidad.

5. Evaluación: es el espacio donde identificamos lo que hemos aprendido a través de una evaluación de las competencias de lectura. En él revisaremos el nivel de comprensión y análisis de los temas tratados en esta reunión.

6. Reflexión: aquí podría interactuar con otros participantes y resolver preguntas relacionadas con la dinámica de la unidad estudiada.

7. La tercera y última parte es la despedida, se realiza la evaluación final que permite evidenciar la movilización de procesos metacognitivos, a través de la cumplimentación del mismo cuestionario (post-test).

\subsection{Procedimiento}

Los participantes fueron asignados de la siguiente manera: el noveno grupo se inscribió en el curso de estudio y el grupo $9 \mathrm{~b}$ en el curso de control, antes de comenzar el curso se les aplicó la prueba ETF, cada estudiante realizó la prueba de forma independiente y de forma autónoma, fue necesaria una sesión grupal con un total de 2 sesiones, esta se desarrolló a partir de un software multimedia que se copió en cada una de las computadoras utilizadas, la profesora e investigadora Paola Intencia estuvo presente durante la aplicación de este instrumento.

Posteriormente, cada uno de los grupos interactúa independientemente con el curso en los espacios académicos del área de ciencias naturales, el tiempo de interacción con la REDA fue de 30 horas distribuidas en tres semanas.

A cada estudiante se le asignó un usuario y una contraseña para ingresar a la plataforma Moodle, al principio, antes de la interacción con el entorno computacional, el estudiante realizó un cuestionario ESCOLA (prueba previa) para demostrar el estado inicial de cada estudiante en el recuento En su proceso metacognitivo, al final de cada unidad de aprendizaje, los estudiantes realizaron una evaluación, además de 2 pruebas intermedias de comprensión de lectura sobre el tema de estudio, cada uno de los estudiantes realizó un total de 9 pruebas Temas 
discriminados en 2 ejercicios y una prueba verificable del logro del aprendizaje por unidad, que se toma como referencia.

Una vez que se completó la interacción con el entorno computacional, se volvió a aplicar el cuestionario ESCOLA (posterior a la prueba) para determinar el estado final de los procesos metacognitivos de los estudiantes.

Los activadores metacognitivos se presentaron al azar al grupo de estudio al comienzo de cada unidad, al realizar los ejercicios propuestos. El objetivo es determinar los efectos del apoyo metacognitivo en el proceso de comprensión de lectura y en el logro del aprendizaje en torno a las ciencias naturales.

\section{RESULTADOS}

\subsection{Condiciones iniciales}

Utilizando los datos recopilados en la investigación a través de los diferentes instrumentos, se realiza un análisis estadístico descriptivo de las diferentes categorías que apoyan el desarrollo de la capacidad metacognitiva y el nivel de logro del estudiante. En el área de las ciencias naturales, antes de la interacción con el entorno computacional sobre la comprensión lectora y las competencias científicas. Ante la capacidad metacognitiva, se analizan los resultados obtenidos en la primera aplicación del Cuestionario ESCOLA (pretest). En cuanto al nivel de logro, se describen las notas obtenidas previamente por los estudiantes en la asignatura de ciencias naturales.

\subsection{Habilidades metacognitivas iniciales en la comprensión lectora}

El cuestionario ESCOLA (escala de conocimiento de lectura) fue respondido por los estudiantes individualmente antes de la interacción con el entorno computacional (pretest). Los resultados arrojados se presentan a continuación.

La tabla 2 muestra el promedio general de la escala de conciencia de lectura, donde la media corresponde a 95.00 y la desviación estándar corresponde (DE) a 6.58. El puntaje mínimo fue 81 y el máximo fue 106 puntos de 168 posibles.

Tabla 2. Estadística descriptiva de la escala de conciencia de lectura. ESCOLA (Pretest).

\begin{tabular}{|c|c|}
\hline N Válido & 19 \\
\hline Perdió & 00 \\
\hline $\begin{array}{c}\text { Medios de } \\
\text { comunicación }\end{array}$ & 95,00 \\
\hline Desviación Estándar & 6.583 \\
\hline Mínimo & 81 \\
\hline Máximo & 106 \\
\hline
\end{tabular}

Fuente: elaboración propia. 


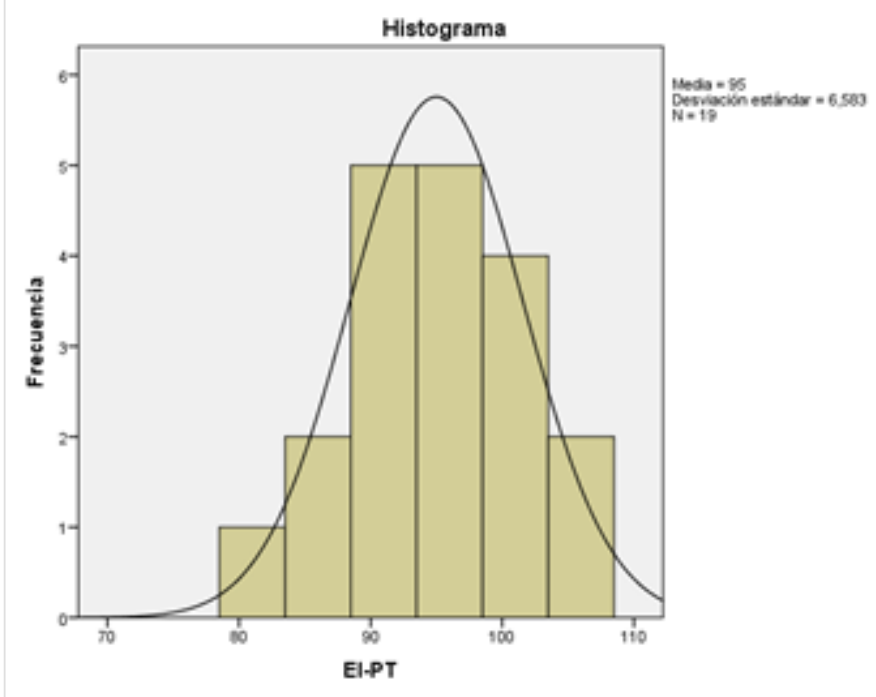

Gráfico 2: Cuestionario de histograma de puntuación. ESCOLA (Pretest).

Fuente: activador metacognitivo inicial (realizado por el autor para la AABW).

El promedio de cada uno de los procesos metacognitivos correspondientes al cuestionario y ESCOLA se indica de forma individual. Los procesos metacognitivos promedio en DIC comieron la presencia de capacidades metacognitivas medianas por los estudiantes.

\subsection{Logros de aprendizaje anteriores}

Las notas obtenidas previamente por los estudiantes en la asignatura de ciencias naturales se consideraron para el análisis de los efectos del apoyo y se describirán a continuación.

Al profesor del área de ciencias naturales se le pidieron las notas obtenidas por los alumnos correspondientes al promedio de dos evaluaciones de competencia científica realizadas durante el primer semestre del año académico. Los estudiantes son evaluados en una escala numérica del 1 al 10. El promedio de las notas es 5.21; Con una desviación estándar (OF) de 1.47. Con un puntaje máximo de 10, el valor mínimo fue 3 y el máximo de 7.

Tabla 3. Estadística descriptiva de la escala de conciencia de lectura. ESCOLA (Pretest).

\begin{tabular}{|l|l|}
\hline $\mathrm{N} \quad$ Válido & 19 \\
\hline \multicolumn{1}{|c|}{ Perdió } & 0 \\
\hline $\begin{array}{l}\text { Medios de } \\
\text { comunicación }\end{array}$ & 5,21 \\
\hline Desviación Estándar & 1,475 \\
\hline Mínimo & 3 \\
\hline Máximo & 7 \\
\hline
\end{tabular}

Fuente: elaboración propia. 
El gráfico 3 muestra que 4 de los estudiantes son evaluados con una nota de 3 que corresponde al $21.1 \%, 1$ de los estudiantes es evaluado con una nota de 4 que corresponde al 5.3\%, 6 estudiantes son evaluados con una nota de 5 correspondiente a $31.6 \%, 3$ de los estudiantes obtuvieron una nota de 6 correspondiente a $15.8 \%$ y 5 de los participantes en el estudio lograron una nota de 7 que representa $26.3 \%$. Se observa en la figura que el desempeño en las evaluaciones no describe una distribución normal.

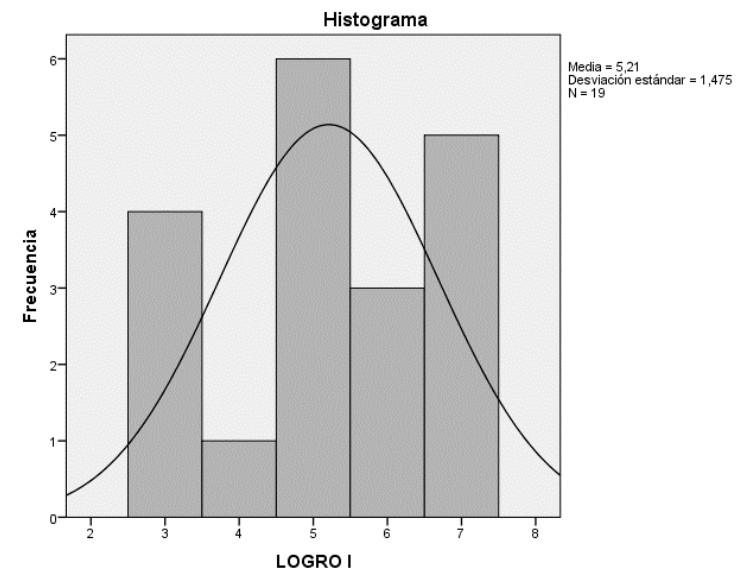

Gráfico 3: histograma de notas Prueba de Ciencias Naturales 2015.

Fuente: imagen de CA de la unidad (realizada por el autor para la AABW).

\subsection{Análisis del efecto del programa}

Para estudiar el efecto del software en sus dos modalidades sobre los procesos metacognitivos y sus diferentes variables y sobre el logro del aprendizaje, se realizó un análisis multivalente de covarianza (Mancova). Para este análisis, las variables dependientes del estudio fueron 1] la capacidad metacognitiva (planificación, supervisión, evaluación y buen lector) y 2] el rendimiento académico (promedio de las evaluaciones de cada unidad). Se consideran dos independientes en el análisis; 1] Trabajar con el entorno computacional que diferencia a los estudiantes que trabajaron en presencia o ausencia de apoyo metacognitivo y 2] el estilo cognitivo (dependiente, independiente e independiente). El análisis de Mancova se realiza tomando como Covariables 1] Los datos iniciales de la capacidad metacognitiva de los estudiantes, la prueba preliminar de ESCOLA (obtenida por la aplicación inicial del cuestionario Escola) y 2] las notas obtenidas previamente en el área de Ciencias Naturales.

En primera instancia, se analiza la descripción detallada de las variables dependientes para el caso; La capacidad metacognitiva (planificación, supervisión, evaluación y buen lector) y el logro del aprendizaje (promedio de las evaluaciones de cada unidad).

\subsection{Las variables dependientes (postest Escola)}

Al final de la interacción con el entorno computacional, los estudiantes respondieron nuevamente el cuestionario ESCOLA. La tabla 4, la de correlaciones, 
presenta las correlaciones de los diferentes procesos metacognitivos; Planificación, seguimiento, evaluación y buen lector y las variables correspondientes.

Esto permite observar el perfil metacognitivo de los estudiantes una vez que se ha completado la interacción con el entorno computacional. Los resultados arrojados se presentan a continuación.

Se muestra el promedio general de la escala de conciencia de lectura, donde la media corresponde a 124.74 y la desviación estándar corresponde (DE) a 31.6. El puntaje mínimo fue 82 y el máximo fue 163 puntos de 168 posibles.

Tabla 4. Estadística descriptiva de la escala de conciencia lectora. ESCOLA (Postest).

\begin{tabular}{|l|l|}
\hline $\mathrm{N} \quad$ Válido & 19 \\
\hline \multicolumn{1}{|c|}{ Perdió } & 0 \\
\hline $\begin{array}{l}\text { Medios de } \\
\text { comunicación }\end{array}$ & 124,74 \\
\hline Desviación Estándar & 31,610 \\
\hline Mínimo & 82 \\
\hline Máximo & 163 \\
\hline
\end{tabular}

Fuente: elaboración propia.

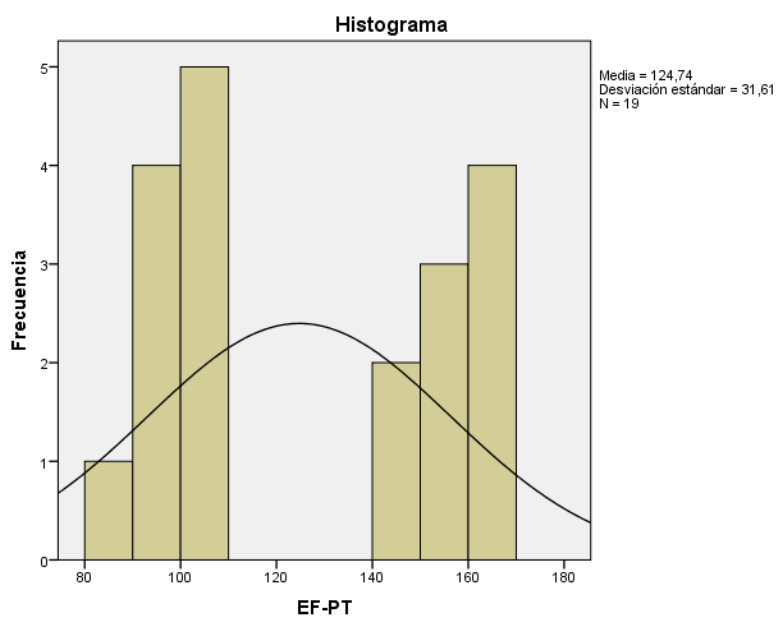

Gráfico 4: Cuestionario de histograma de puntuación ESCOLA. (Posttest).

Fuente: activador de prequiz (realizado por el autor para la AABW).

Los procesos metacognitivos promedio en DIC comieron un aumento en las capacidades metacognitivas de acuerdo con los resultados de la aplicación inicial.

\subsection{Logro de aprendizaje de ciencias naturales}

Es importante recordar que el logro del aprendizaje se evaluó individualmente al final de la interacción con cada una de las unidades que constituyen el entorno computacional. El logro general se obtuvo del promedio de los exámenes 
presentados por los estudiantes. El promedio del logro general corresponde a 7.30 con una desviación estándar (OF) de 1.95. El valor mínimo es 4 y el máximo es 10, lo que representa los resultados de las tres pruebas en la nota máxima posible.

Tabla 5. Estadística descriptiva del logro del aprendizaje general en ciencias naturales.

\begin{tabular}{|l|l|}
\hline N Válido & 19 \\
\hline \multicolumn{1}{|c|}{ Perdió } & 0 \\
\hline $\begin{array}{l}\text { Medios de } \\
\text { comunicación }\end{array}$ & 7,30 \\
\hline Desviación Estándar & 1,953 \\
\hline Mínimo & 4 \\
\hline Máximo & 10 \\
\hline
\end{tabular}

Fuente: elaboración propia.

A continuación se presenta la distribución de las notas obtenidas por cada uno de los estudiantes que participaron en el estudio.

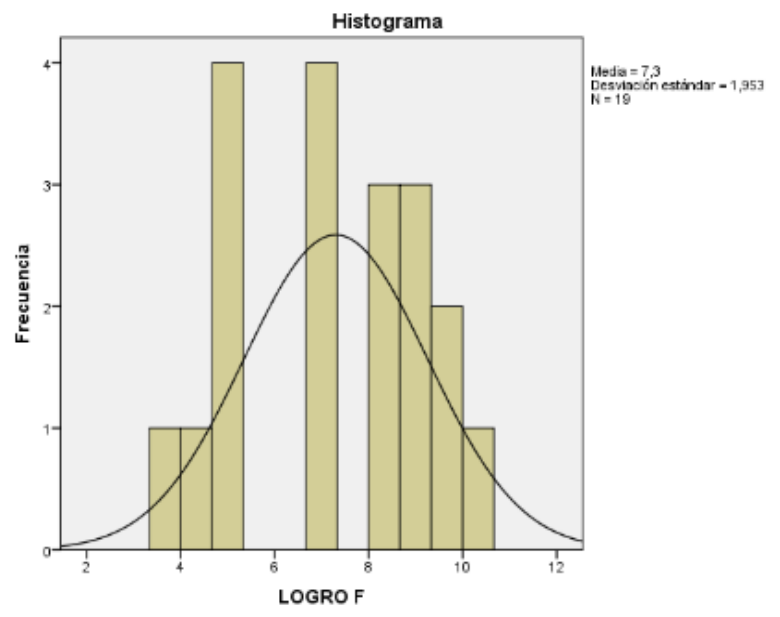

Gráfico 5: histograma de logros generales de aprendizaje en ciencias naturales. Fuente: elaboración propia.

\subsection{Análisis multivariante (Mancova)}

Los resultados de Mancova muestran que los modelos resultantes tienen un alto nivel de predicción de las diferentes variables dependientes incluidas. Sin duda, una de las variables que alcanza una mayor explicación de su varianza es el logro del aprendizaje, que se alcanza para predecir el $98.1 \%$ de la varianza $(R 2=0,981)$.

En cuanto a las diferentes variables del cuestionario ESCOLA podemos encontrar relaciones significativas de forma generalizada siendo el proceso de evaluación en la persona variable con el $99.3 \%$ de la varianza $(R 2=0,993)$ la más alta; seguido de la planificación en la categoría de persona (persona con FP) con el $98.7 \%$ de la varianza ( $R 2=0,987)$, seguido de un buen lector (lector BF) con el $98.5 \%$ de la 
varianza ( $R 2=0,985)$, seguido del logro de aprendizaje mencionado anteriormente ; Posteriormente, el proceso de monitoreo se informa en las variables de tarea y texto (Tarea SF) y (texto SF) respectivamente con el $97 \%$ de la varianza (R2=0,97), en forma consecutiva el proceso de evaluación final se informa en la variable de tarea con El $96.8 \%$ de la varianza (R2=0,968), seguido del proceso de planificación final en la variable de tarea (tarea PF) con el $96,7 \%$ de la varianza $(R 2=0,967)$, al final se encuentra, el proceso de monitoreo en la variable persona ( SF persona) con $93 \%$ de la varianza $(R 2=0,930)$. Al medir el análisis de las relaciones entre las covariables y las variables, los resultados muestran que cada una de las covariables muestra una asociación significativa, especialmente con el estado final de la misma variable dependiente $y$, en algunos casos, con otras variables.

En el caso de la planificación en la variable persona, se establecen relaciones significativas con este mismo proceso en la misma variable con ( $F=23,39 ; p=0,008)$ y con un valor ligeramente inferior con el proceso de evaluación final en la variable tarea con $(F=8,15 ; p=0,046)$.

Por otro lado, el proceso de planificación en la variable de tarea establece relaciones significativas con los procesos de planificación en la persona y tarea de la variable con $(F=19,51 ; p=0,012)$ y $(F=6,91 ; p=0,058)$ respectivamente. Para el proceso de monitoreo en la variable persona solo se establece una relación significativa con el mismo proceso en la misma variable con $(F=9,03 ; p=0,040)$. En cuanto al proceso de supervisión inicial del texto, esto establece relaciones significativas con el proceso de planificación final de la persona con $(F=10,28$; $p=0,033$ ). Por otro lado, el proceso de evaluación inicial en la variable persona logró establecer diferentes relaciones consideradas significativas; En primera instancia, encontramos el mismo proceso en la misma variable con $(F=24,07 ; p=0,008)$, seguido del proceso de planificación final en la persona variable con a $(F=15,13 ; p$ $=0,018)$ y termina con el logro del aprendizaje donde se informa uno $(F=8,07$; $\mathrm{p}=0,047)$.

Frente a las estrategias condensadas en la categoría de buen lector, este informa relaciones significativas con tres variables en primer lugar con el mismo proceso en la misma variable con $(F=15,11 \mathrm{p}=0,018)$, seguido por el logro del aprendizaje con $(F=8,71 \mathrm{p}=0,042)$, y finalmente, el proceso de monitoreo final se informa en la variable de tarea con $(F=7,32 p=0,054)$.

Por otro lado, la variable de grupo establece relaciones significativas con todas las categorías de la ESCOLA, siendo estas altamente significativas.

En relación con los efectos principales, el efecto más significativo está dado por la presencia de apoyo metacognitivo, ya que los resultados muestran una asociación importante con el logro del aprendizaje. Una simple encuesta de datos revela que los estudiantes que trabajaron en el entorno con apoyo metacognitivo mostraron resultados mucho más altos que sus compañeros que trabajaron en el entorno sin apoyo metacognitivo. Del mismo modo, es evidente que la presencia de apoyo 
metacognitivo favoreció el aumento de las habilidades metacognitivas, es decir, los procesos de planificación, monitoreo y evaluación.

Por otro lado, la variable "el estilo cognitivo en la dimensión DFI" no evidencia ningún asociado significativo, lo que lleva a suponer que el apoyo minimiza las diferencias previamente presentes y dadas por las características del estilo cognitivo de los estudiantes.

\subsection{Limitaciones}

Hay varias limitaciones que deben considerarse para la interpretación de sus conclusiones. Se pueden mencionar tres limitaciones importantes.

En primera instancia podemos mencionar ese sentido estricto, el estudio no fue una investigación experimental, que incluyó todos los controles de rigor, sino que tuvo que limitarse a una situación experimental con dos grupos previamente formados de noveno grado en el espacio académico de Natural Ciencias en una escuela privada de la ciudad de Bogotá. Por esta razón, no es posible generalizar los resultados a todos los estudiantes de secundaria en nuestro sistema educativo. Sin embargo, la investigación deja un espacio abierto que permite a otros investigadores continuar realizando estudios en diferentes dominios de conocimiento y niveles académicos.

Por otro lado, se destaca que el instrumento utilizado para medir el nivel de conciencia de lectura alcanzado por los estudiantes de secundaria, ya que para esto se utilizó un informe de autor del cuestionario (escala de conciencia de lectura, ESCOLA), ampliamente utilizado en esta área de investigación. Por esta razón, existe la probabilidad de que exista un sesgo incontrolable dado por la tendencia de los estudiantes a dar respuestas socialmente aceptadas, un factor que la investigación no pudo controlar.

Finalmente, es importante referirse al tamaño de la muestra porque cuando subdividen los grupos por todas las variables independientes, esto es muy pequeño. Para experiencias posteriores, se sugiere aumentar el tamaño de la muestra.

\subsection{Recomendaciones para futuras investigaciones}

Para futuras investigaciones sobre el tema, es importante tener en cuenta en un nivel más amplio de estudio las características del texto como elemento fundamental en la comprensión lectora, de la misma manera es importante extender el tamaño de la muestra para que el Los resultados pueden generalizarse, ya que sería interesante ampliar las unidades de aprendizaje propuestas para el medio ambiente porque, aunque la investigación mostró resultados importantes, los cambios en los niveles metacognitivos implican interacciones representativas con el medio ambiente.

Por otro lado, sería interesante llevar a cabo la investigación con la integración del componente de comprensión lectora y las áreas de ciencias exactas y naturales para 
lograr inferencia y promover el desempeño de los estudiantes en pruebas estandarizadas a través de la web en entornos de aprendizaje basados.

\section{CONCLUSIÓN}

La presente investigación contribuye al campo del diseño de entornos de aprendizaje basados en la Web AABW de la siguiente manera:

Según la teoría especializada en el campo de las tecnologías de la información aplicadas a la educación, se retoma la importancia del diseño de andamios metacognitivos para fortalecer las posibilidades de monitoreo y regulación en el proceso de Aprendizaje y de esta manera fomentar la comprensión lectora y, por lo tanto, logro del aprendizaje. Para los propósitos de este estudio, los datos proporcionados por los estudiantes involucrados en el proceso de investigación muestran que un apoyo metacognitivo incluido en un entorno basado en la WEB aumenta sus habilidades metacognitivas y, en consecuencia, el logro del aprendizaje en las pruebas de comprensión lectora de textos científicos.

Por otro lado, es importante enfatizar la técnica estadística de alta complejidad que se utilizó en la presente investigación para determinar la interacción de las variables. Utiliza múltiples instrumentos y técnicas estadísticas robustas, como el análisis de covarianza Mancova, para estudiar el impacto de variables independientes además de controlar diferentes variables asociadas que pueden estar relacionadas con variables dependientes. Este análisis contribuye a dar al estudio y a los protagonistas del campo educativo una mayor validez y fiabilidad.

Otro elemento importante que contribuye significativamente al contexto educativo se refiere a la población, ya que la mayoría de los estudios $D$ toman como referencia a la población universitaria, la presente investigación centró su atención en la población de secundaria. Mostrando evidencia de cómo juegan las estrategias metacognitivas en la comprensión lectora. Un papel fundamental en la realización de pruebas estandarizadas en el área de las ciencias naturales en entornos de aprendizaje basados en la web $y$, a su vez, afecta la presencia de un apoyo metacognitivo en estas variables.

La investigación proporciona información relevante sobre la importancia de diseñar e implementar entornos de aprendizaje basados en la web con andamios que favorezcan el desarrollo de habilidades metacognitivas. Teniendo en cuenta los diferentes procesos y variables que intervienen cuando el sujeto se enfrenta a una lectura de tipo científico. Del mismo modo, este contexto proporciona herramientas para la realización del control y seguimiento del proceso de aprendizaje de los estudiantes. Esta investigación es de mayor interés para la comunidad educativa del país porque el uso de entornos virtuales de aprendizaje está muy extendido y progresivamente se vuelve más cercano e indispensable para el trabajo educativo, por lo que es necesario promover estrategias didácticas como el apoyo informático para obtener un mayor aprendizaje y un mejor desempeño en las diferentes áreas de conocimiento en la población estudiantil de secundaria. 


\section{REFERENCIAS}

Alonso, C., Gallegos, D., y Honey, P. (1997). Los estilos de aprendizaje. Bilbao, España: Mensajero.

Cantillo, K., De la Hoz , Á. y Cerchiaro , E. (2014). Actividad metacognitiva en estudiantes universitarios: un estudio preliminar. Psicología desde el Caribe, 31(3), 455-474.

Cerchiaro, E., Paba, C. y Sánchez, L. (2011). Metacognición y comprención lectora: una relación posible e intencional. Revista de la Facultad de Ciencias de la Salud, Universidad del Magdalena, 8(1), 99-111.

De Corte, E. (1999). Una concepción del aprendizaje y la enseñanza para el siglo XXI. Revista Latina de Pensamiento y Lenguaje, (4), 251-278.

Gallego Torres, R. (2017). Study telling, base teórica de una nueva comunicación para el e-learning. In Caleidoscopio Educativo (pp. 135-144). Madrid: Global Knowledge Academics.

Gallego, \& Honey (1997). Los estilos de aprendizaje. Bilbao: Mensajero.

García, D. (2015). Una estrategia didáctica en los ambientes de aprendizaje basados en la web: Andamiaje autorregulador. $V$ Encuentro Internacional de Gestión del Conocimiento: Gestión de Investigación. Girardot.

Gundín, O., Fidalgo, R. y Robledo, P. (2012). Metacognición y comprensión lectora: conocimiento y uso de estrategias. International Journal of Developmental and Educational Psychology, 2(1), 195-201.

Hederich-Martínez, C. (2007). El estilo cognitivo en la dimensión de dependencia e independencia de campo. Influencias culturales e implicaciones para la educación. Bogotá: Universidad Nacional de Colombia.

Hederich-Martínez, C. y Camargo-Uribe, Á. (2018). Estilo cognitivo y logro académico en la ciudad de Bogotá. Educ.Educ, 40-41.

Hederich, C. (2013). Estilística educativa. Revista Colombiana de Educación, (64), 2156.

Hermann, A., \& Detlev, L. (1995). Learning Strategies for Unstructured Hypermediaa Framework for Theory, Research, and Practice. Journal of Educational Computing Research, 13(4), 387-400.

Herrero, C., \& Brown, M. (2010). Distributed Cognition. Revista de Psicodidáctica, $15(2), 253-268$. 
Hidalgo , A., \& Olaya, L. (2016). Educational stylistics and activities for language development at the preschool level in two public schools of Bogota, Colombia. Revista de Estilos de Aprendizaje, 9(17), 25-53.

ICFES. (2013). Colombia en Pisa 2012, Informe Nacional de resultados. Bogotá: ICFES.

Jiménez, V. (2004). Metacognición y comprensión de lectura. Evaluación de los componentes estrategicos mediante la elaboración de una escala de conciencia lectora. Madrid: Universidad Complutense.

Korthauer, R., \& Koubek, R. (2009). An empirical evaluation of knowledge, cognitive style, and structure upon the performance of a hypertext task. International Journal of Human-Computer Interaction, 6(4), 373-390.

López, Hederich \& Camargo (2011). Estilo cognitivo y logro académico. Educación y Educadores, 67-82.

López, Ó. (2008). Estilo Cognitivo y Diseño de Ambientes Computacionales. Memorias del VII Congreso de Informatica Educativa - RIBIE. Barranquilla.

López, O., Ibáñez, J. y Chiguasuque, E. (2014). El estilo cognitivo y la fijación de metas deaprendizaje en ambientes computacionales. Pensamiento Psicológico, $12(1), 133-148$.

López-Vargas, Ó. y Hederich-Martínez, C. (2011). Aprendizaje autorregulado, estilo cognitivo y logro académico en ambientes computacionales. Revista Colombiana de Educación, 165-172.

Lozano (2006). Estilos de aprendizaje y enseñanza: Un programa de la estilística educativa. México: Trillas.

Min, L., \& Reed, M. (1994). The relationship between the learning strategies and learning styles in a hypermedia environment. Computers in Human Behavior, 10(4), 419-434.

Molenaar, I., van Boxtel, C., \& Sleegers, P. (2010). The efects of scaffolding metacognitive in smoll group. Elsevier.

OCDE (2016). Programme for internacional student asseement. Results from PISA 2015. OCDE.

Paba , C. y González, R. (2014). La actividad metacognitiva y la comprensión lectora en estudiantes de décimo grado. Psicología desde el Caribe, 31(1), 79-101.

Renes, P. y Martínez, P. (2016). Una mirada a los estilos de enseñanza en función de los estilos de aprendizaje. Revista de Estilos de Aprendizaje, 9(18), 224-243. 
Diseño, producción e implementación del ambiente de aprendizaje basado en web, AABW

Sierra, I. (2010). Estrategias de mediación metacognitiva en ambientes convencionales y virtuales: influencia en los procesos de autorregulación y aprendizaje autónomo en estudiantes universitarios. Granada: Universidad de Granada.

Sierra, I. y Carrascal, N. (2011). Concepciones y estrategias docentes en entornos virtuales, fundamentos para una tipologia. Monteria: Universidad de Córdoba.

Sierra, I., Carrascal, N. y Buelvas, R. (2014). Los entornos tecnológicos con orientación metacognitiva como recursos para la gestión de ambientes y redes personales de aprendizaje en educación superior. Congreso Iberoamericano de Ciencia, Tecnología, Innovación y Educación. Buenos Aires.

Spiro, R., \& Jehng, J.-C. (1992). Cognitive flexibility and hypertest: Theory and technology for the nonlinear and multidimensional traversal of complex subject matter. In Cognition, Education, and Multimedia, Exploring Ideas in High Technology (pp. 163-205). Hillsdale: Lawrence Erulaum Associates.

Tamayo Alzate, O., Zona López , J. y Loaiza Zuluaga, Y. (2016). La metacognición como constituyente del pensamiento crítico en el aula de ciencias. Revista Tecné, Episteme y Didaxis: TED, 1031-1036.

Tergan, S. O. (1997). Conceptual and Methodological Shortcomings in Hypertext/Hypermedia Design and Research. Journal of Educational Computing Research, 16(3), 209-235.

Van de Pol, J., Volman, M., Oort, F., \& Beishuizen, J. (2015). The effects of scaffolding in the classroom: support. Instr Sci, 615-641. doi: https://doi.org/10.1007/s11251-015-9351-z

\section{AUTOR:}

\section{Rómulo Andrés Gallego Torres}

Profesor investigador y evaluador sobre temas como e-learning, comunicación digital, nuevos modelos educomunicativos, nuevas narrativas digitales y Digital StoryTelling. Experto profesional en el desarrollo de soluciones digitales, diseñador gráfico y productor de UNITEC, comunicador social de la Fundación Universitaria San Alfonso, maestro en Tecnología de la Información aplicada a la educación en la Universidad Pedagógica Nacional, PhD en Educación; escuela, idioma y sociedad de la Universidad del País Vasco, fundador de Comunicación Visual - Taller creativo, amante de la lectura sobre temas relacionados con las TIC, la educación y las ciencias sociales y convencido de que el acceso democrático a la educación tiene su propia línea de desarrollo en la adecuada uso de nuevas tecnologías.

andresgallegot@gmail.com

Orcid ID: https://orcid.org/0000-0001-5074-6781 